\title{
$\$$ Research Square

\section{Machine Learning for the Prediction of Progression in Patients With Acute Kidney Injury in Critical Care}

\section{Lifan Zhang}

Sichuan University West China Hospital https://orcid.org/0000-0001-5182-3814

\section{Canzheng Wei}

Sichuan University West China Hospital

Xuepeng Zhang

Sichuan University West China Hospital

Aijia Ma

Sichuan University West China Hospital

Jiangli Cheng

Sichuan University West China Hospital

\section{Meiling Dong}

Sichuan University West China Hospital

Jing Yang

Sichuan University West China Hospital

Yan Kang ( $\sim$ Kangyan@scu.edu.cn )

Sichuan University West China Hospital https://orcid.org/0000-0003-4968-1217

\section{Research}

Keywords: Acute kidney injury, Critical care, Extreme gradient boosting

Posted Date: November 12th, 2020

DOI: https://doi.org/10.21203/rs.3.rs-102978/v1

License: (c) (i) This work is licensed under a Creative Commons Attribution 4.0 International License.

Read Full License 


\title{
RESEARCH
}

\section{Machine learning for the prediction of progression in patients with acute kidney injury in critical care}

Lifan Zhang ${ }^{1}$

, Canzheng Wei ${ }^{2}$

, Xuepeng Zhang ${ }^{2}$

, Aijia $\mathrm{Ma}^{2}$

, Jiangli Cheng ${ }^{2}$

, Meiling Dong 2

, Jing Yang ${ }^{2 \dagger}$

and Yan Kang ${ }^{2 * \dagger}$

${ }^{*}$ Correspondence:

Kangyan@scu.edu.cn

${ }^{2}$ Department of Critical Care

Medicine, West China Hospital of

Sichuan University, 610041

Chengdu, China

Full list of author information is

available at the end of the article

t These authors contributed to the

work equally and should be

regarded as co-corresponding

authors.

\begin{abstract}
Background: Acute kidney injury (AKI) is a serve and harmful syndrome in the intensive care unit. Our previous study has shown that patients who will progress to AKI 3 stage are considered to receive RRT. This study aimed to develop a prediction model that can predict whether progression to AKI stage 3 .

Methods: Patients with AKI stage $1 / 2$, when they were first diagnosed with AKI in the Medical Information Mart for Intensive Care (MIMIC-III), were included. Patients who receive RRT or progress to AKI 3 stage within 72 hours of first AKI diagnosis were excluded. We build two predictive models, respectively using machine learning extreme gradient boosting (XGBoost) and logistic regression, to predict patients who will progress to AKI stage 3. Established models were evaluated by cross-validation and area under receiver operating characteristic curve (AU-ROC).
\end{abstract}

Results: Of the 29238 patients included in the analysis, 3237 (11.1\%) patients progressed to AKI stage 3. Creatinine, blood urea nitrogen (BUN), sepsis and respiratory failure were the important predictors of $A K I$ progression. The machine learning XGBoost model has a better performance than the Cox regression model on predicting $\mathrm{AKI}$ stage 3 progression (AU-ROC, 0.860 vs. 0.728 , respectively).

Conclusions: The XGBoost model was able to identify patients with AKI progression better than the Cox regression model. Machine learning techniques may improve predictive modeling in medical research.

Keywords: Acute kidney injury; Critical care; Extreme gradient boosting

\section{Introduction}

Acute kidney injury (AKI) is a common syndrome in the intensive care unit defined by an abrupt increase in serum creatinine and a decrease in urine output[1]. AKI can be attributable to simple causes such as dehydration and the use of nephrotoxic drugs or multiple causes. The course of AKI can be variable, from complete recovery to chronic kidney disease or end-stage renal disease[2] Renal replacement therapy (RRT) is the mainstay of treatment for advanced AKI, but RRT is possibly harmful and not available in all settings and regions. In our previous studies, we found that 
only the critical ill patients progress to AKI stage 3 could benefit from RRT therapy. Those who high risk to progress are considered to receive RRT as soon as possible. Under this circumstance, the prediction of whether progression to AKI stage 3 is urgent and crucial.

Clinicians have not enough tools to predict whether AKI stage $1 / 2$ patients will progress to more severe stages. Chawla LS et al. investigated the furosemide stress test (FST) as a method with the robust predictive capacity to identify those who will progress to AKI stage 3[3] This biomarker was significantly better than other common urinary biomarkers such as plasma neutrophil gelatinase-associated lipocalin and urinary Interleukin - 18 on predicting progression to AKI stage 3. However, FST was found to have numerous limitations and was not commonly used in clinical application. Not all patients are stable for furosemide challenge and other factors such as fluid balance and diuretic have an ambiguous effect on the outcome.

Currently, several studies are proposing a prediction model on identifying patients' AKI progression. But the development and validation of these prediction models are not desirable. And the clinical application has been hampered for several reasons such as few general hospital population studies. With the development of processing power, memory and storage, computers can tackle increasingly complex learning tasks, which is so-called machine learning. There are thousands of papers applying machine learning algorithms to medical data in recent years [4].

Therefore, we hypothesized that machine learning techniques may be useful to build a robust predictive model. In this study, we collected data from the database to predict AKI stage 3 progression by machine learning techniques(extreme gradient boosting) and logistic regression, and compared the performance of these two models.

\section{Method}

Source of data

MIMIC-III (Medical Information Mart for Intensive Care III) is a large, deidentified, comprehensive dataset associated with patients who admitted to the ICUs of the Beth Israel Deaconess Medical Center in Boston, MA, between 2001 and 2012[5] This database includes demographics, vital sign measurements, laboratory test results, and so on. Since the study was an analysis of a third-party anonymized publicly available database with pre-existing institutional review board (IRB) approval, IRB approval from our institution was exempted.

\section{Participants}

AKI was defined as an increase in serum creatinine of $0.3 \mathrm{mg} / \mathrm{dl}$ or $50 \%$ from the baseline value or urine output $<0.5 \mathrm{ml} / \mathrm{kg}^{*} \mathrm{~h}[1]$ This definition was consistent with the serum creatinine and urine output components of the Kidney Disease Improving Global Outcomes (KDIGO) criteria. The critically ill patients were included if their primary diagnosis was AKI stage $1 / 2$. We included patients as more as possible to improve the statistical power of the predictive model. Patients who are younger than 18 years old were excluded. Furthermore, patients who received RRT or progressed to AKI stage 3 within 72 hours of first AKI diagnosis were excluded. The low rate of progression patients may have bad effect on model generalization. 
However, a combination of oversampling and undersample[6] can balance the proportion of patients in the two groups, which can limit the impact on the outcome to a minimum.

\section{Predictors of Model}

We collected clinical and laboratory variables obtained within 72 hours before and after the first AKI diagnosis. For some variables with multiple measurements in these 6 days, we define the closest measurement as we need. We analyzed age and vital signs including respiratory rate, blood pressure, heart rate, and temperature. Besides, laboratory data including glucose, potassium, sodium, lactate, creatinine, blood urea nitrogen (BUN), anion gap, $\mathrm{PaO} 2$, AaDO2, and $\mathrm{pH}$ were included as well[7]. Furthermore, we also analyzed participants whether received vasoactive drugs, cardiac surgery, mechanical ventilation, and whether have sepsis, respiratory failure, and multiple organ failure syndromes (MODS)[8]

Specifically, creatinine was calculated the mean of measurement within 6 days because serial measurements have better predictive capability than single timepoint [9]. Missing data may create bias, so variables with more than $70 \%$ missing values were excluded. Extreme gradient boosting (XGBoost) can automatically process missing values. As for the Cox regression model, we complete missing values using the multiple imputation method in scikit-learn[10].

\section{Model Selection and Development}

We compared characteristics between groups by Student t-test. In addition, as for categorical and nonmoral variables, we used the Chi-square test and the KruskalWallis Rank Sum Test respectively.

We used the Cox regression model to select predictive variables for AKI progression. The proportional hazard auusmption is not necessary to process as our study's goal is to develop predict model for AKI stage 3[11]. Both forward selection and backward elimination were used, scoring at each step for variables to be included or excluded. Akaike Information Criterion (AIC) was used to evaluate the predictive capability. The lower AIC, the better predictive performance XGBoost of the model.

Extreme gradient boosting (XGBoost) is an ensemble method of machine learning based on decision trees[12] We set a decision tree as the weak learner and the learning objective as binary logistic. In this method, we iteratively refitting a weak classifier (decision tree) to residuals of previous models. Each classifier focused more on misclassified observations during the previous rounds. XGBoost hyperparameters included learning rate, minimum child weight, maximum depth of a tree and subsample ratio, and so on. In this study, we employed 100 rounds of iterations for the cross-validation process, which is also the default and recommended value[13].All analyses were performed using Python, version 3.7.9.

To estimate the predictive capability, we used cross-validation. The original data was randomly divided into 5 equal-sized subsamples. Four subsamples were used to train the model, which was then validated in the remaining one subsample. Hyperparameters were considered to be successfully tuned when the training log-loss decreased as the number of rounds increased and the testing log-loss was less than 
0.693 and only slightly greater than the training log-loss. Because a binary classifier that performs equally with chance have a log-loss of 0.693 . On this basis, hyperparameters were tuned for the higher area under receiver operating characteristic curve (AU-ROC) which was used to evaluate the predictive ability of the model.

We used grid search which can cycle through tuning and scoring to select the hyperparameters. After choosing the hyperparameters, the XGBoost was trained for the final model. During the training process, overfitting or underfitting may be problems in using machine learning techniques. The ability to understand the complex relationship in data while avoiding overfitting requires fine-tuned hyperparameters. Furthermore, we calculate AU-ROC by changing the subsample ratio to draw the learning curve which can also help us detect overfitting and underfitting.

\section{Results}

Participants

Of the 61532 patients in MIMIC-III, 34438(56.0\%) patients were diagnosed AKI stage $1 / 2$ of the first AKI diagnosis. A number of 6990 patients were excluded for some reasons. A total of 27448 patients were included in our analysis; 3119(11.4\%) patients finally progress to AKI stage 3, and 24329(88.6\%) patients did not (Fig. 1). After balance of patients in two groups, there are 38842 observations in total, $23360(60.1 \%)$ observations with progression, and 15482(40.9\%) observations without progression.

The differences in characteristics between groups are described in Table 1. The progression group had fewer patients who received cardiac surgery than the nonprogression group $(72.3 \%$ vs. $80.9 \% ; p<0.001)$. The creatinine $(2.9 \pm 2.4$ vs. $1.3 \pm$ $1.3 \mathrm{mg} / \mathrm{dl} ; p<0.001)$ and the BUN $(41.3 \pm 27.1$ vs. $24.5 \pm 18.3 \mathrm{mg} / \mathrm{dl} ; p<0.001)$ were higher, and FST $(158.6 \pm 231.2$ vs. $198.5 \pm 134.3 \mathrm{ml} / \mathrm{h} ; p<0.001)$ was lower in the progression group. The progression group had higher heart rate $(89.4 \pm 20.7$ vs. $85.7 \pm 18.0 ; p<0.001)$, higher lactate $(2.4 \pm 2.1$ vs. $2.0 \pm 1.6 \mathrm{mmol} / 1 ; p<0.001)$, the higher rate of mechanical ventilation ( $43.6 \%$ vs. $29.6 \% ; p<0.001)$, the higher rate of MODS $(95.7 \%$ vs. $60.4 \% ; p<0.001)$, the higher rate of respiratory failure $(60.8 \%$ vs. $33.8 \% ; p<0.001)$ than the non-progression group.

\section{The stepwise Cox regression model}

The results of the stepwise Cox regression model are shown in Table 2 \& Fig. 2. We use original data that are not be balanced in groups to test the model to reflect true performance. As expected, with MODS (odds ratio[OR], 2.90; 95\% confidence interval[14], 2.68 to 3.15 ), sepsis(OR, 1.56; 95\% CI, 1.49 to 1.63), respiratory failure (OR, 1.21; 95\% CI, 1.15 to 1.28), and mechanical ventilation (OR, 1.22; 95\% CI, 1.16 to 1.29 ) were associated with increased probability of AKI progression(Table 1). Besides, creatinine, potassium, and so on are also considered to be associated with AKI progression. On the contrary, a greater value of $\mathrm{pH}(\mathrm{OR}, 0.61 ; 95 \% \mathrm{CI}$, 0.47 to 0.80 ), temperature (OR, $0.84 ; 95 \%$ CI, 0.81 to 0.86 ), and sodium (OR, 0.99; $95 \%$ CI, 0.98 to 0.99 ) were associated with decreased probability of AKI progression (Table 2). 


\section{The XGBoost model}

Determining by grid search, the hyperparameters used in our analysis were set as learning rate $=0.17$, minimum child weight $=6$ maximum tree depth $=3$, and the number of rounds $=100$. With these hyperparameters, the training score increases as the number of rounds increases, and the cross-validation score testing log-loss was less than 0.693 and only slightly more than the training log-loss as the tree grows (Fig. 3A).

In the learning curve, the training score decreases, and the test score increase as subsample ratio increase. And the training score is always higher than the test score (Fig. 3B). In other words, with the simple size expands, the model has a better predictive performance, which suggests there is no overfitting or underfitting. Therefore, this model has good generalization ability and robustness.

\section{Model performance}

The model was scored using the AU-ROC. The XGBoost had a significantly greater AU-ROC than the logistic regression model (AU-ROC, 0.863 vs. 0.776, respectively; Fig. 4A). We also showed the confusion matrix for the two models in predicting AKI progression (Fig. 4B).

\section{Tree interprets}

SHAP (SHapley Additive exPlanations) is a game-theoretic approach to explain the output of the machine learning model[15]. As for this dichotomous classifier, the higher SHAP value, the higher probability of AKI progression. The base value is defined as the average model output over the training dataset, which can represent the average of the sample. With original data, we calculate the base value is -2.23 . Therefore, the average of these patients is unlikely to progress to AKI stage 3, which can be explained by the relatively low proportion of progression patients $(11.4 \%)$.

SHAP value can intuitively show features each contribution to push the model output from the base value (Fig. 5A). SHAP value can be considered as a quantified contribution. We can easily find the contribution of all features and which contribution is most (Fig. 5B). Feature importance was calculated by the mean contribution of every observation, which is equal to the traditional method[16]. The serum creatinine was the most important variable, followed by MODS and respiratory failure. (Fig. 6)

\section{Discussion}

Broadly speaking, machine learning techniques have better performance than the traditional regression model[17]. By Extreme gradient boosting (XGBoost), some vital signs and laboratory factors were found to be associated with AKI progression. In our model, some factors are more important than others such as creatinine and MODS. These results can be applied in the intensive care unit.

First, our results showed that machine learning techniques have better performance than Cox regression. In one way, some factors are found to have a non-linear relationship between value and risk, which cannot be explained by the Cox regression model which is essentially a linear model. In another way, extreme gradient boosting iteratively focusing more on misclassified observations can greatly improve 
performance especially if the number of iterations is large enough. Almost all the other studies showed that machine learning techniques are better than traditional regression models in prediction[18, 19, 20].

Second, this model can identify whether patients will progress to AKI automatically, which provide assessments and recommendations to physicians. Currently, there is still lack of a reliable tools to predict AKI progress and find the major reasons caused. XGBoost can automatically collect data and provide a binary probability for AKI progression as a part of clinical workflow. Therefore, we can get an intuition about the severity of illness. And, with SHAP, it is easy and intuitive to describe the contribution of each variable to the final result (Fig. 5A), which recommend clinicians how to decrease the risk. These features were found to correlate with decision support systems' ability to improve patient care[21, 22]. However, present studies cannot provide a higher level of evidence on the effectiveness or performance of the XGBoost model. Future randomized controlled trials comparing patient prognosis with and without the prediction model are required.

Third, in previous studies of predicting AKI, there are some recognized factors such as age, creatinine, FST[23], and so on. As for age, it was considered to be an important predictor or even an independent risk factor[24]. However, it is not as important as we expect in our model. We employed interaction SHAP value between age and creatinine to find an explanation. In AKI progression, the effect of age on outcomes may be masked by the interaction between other factors and age. Age is an important factor for AKI progression only when creatinine was high. But at the overall level, age had little effect on outcomes (Fig. 7A). Then, SHAP value was found to increase with the increase of creatinine until creatinine probably reach $5 \mathrm{mg} / \mathrm{dl}(440.5 \mu \mathrm{mol} / \mathrm{L})$ (Fig. 7B). This is in line with the mainstream view in clinicians[25, 26]. However, the nonlinear relationship after $5 \mathrm{mg} / \mathrm{dL}$ may be the limitation of the model and requires further research. The relationship between SHAP value and FST is consistent with previous studies that FST $<100 \mathrm{ml} / \mathrm{h}$ increases the risk of AKI progression[3, 27].

Our study has some strengths and weaknesses. The XGBoost modeling is a new technique that has not been widely used in medical research. It has been successfully used in some complex problems such as the prediction of the volume responsiveness in patients with AKI[19], in which the performance of the XGBoost model was significantly better than a traditional linear model. Because the XGBoost model is an ensemble of weak prediction trees, which can capture complex and non-linear relationships. Besides, this technique is well designed to prevent overfitting or underfitting by cross-validation and regularization. It can also automatically process missing values, which is significant for clinical practice in predicting at any time. Furthermore, with SHAP, we can understand how the model work and how to simplify the model.

A limitation of this study is a cross-sectional study requiring further randomized controlled study to verify the conclusion. And, the overall population had low blood pressure $(76.7 \pm 16.1 \mathrm{mmHg})$, higher BUN $(26.3 \pm 20.1 \mathrm{mg} / \mathrm{dl})$, higher lactate $(2.1 \pm 1.7 \mathrm{mmol} / \mathrm{l})$, and higher $\mathrm{PaO} 2(128.3 \pm 77.9 \mathrm{mmHg})$ in our study, which was consistent with AKI progression (Table 1). higher BUN and lactate may be associated with AKI stage 1/2. The high rate of Mechanical ventilation (30.8\%) 
was considered to result in higher PaO2. low blood pressure may be the course of vasoactive drugs use (30.6\%). Furthermore, the study only explored the outcomes that whether patients progressed to AKI stage 3. The time from the first diagnosis to progression varied from 3 days to 90 days. However, when to progress was not investigated and required further studies.

\section{Conclusion}

In conclusion, we found that some vital signs and laboratory factors were associated with AKI progression. The XGBoost model may be a better-performing predictive model than COX to identify patients with AKI progression. The contribution of some variables to the risk of AKI progression is not as linear and simple as we expected.

\section{Abbreviations}

AKI: Acute kidney injury; MIMIC-III: Medical Information Mart for Intensive Care; AU-ROC : area under receiver operating characteristic curve; BUN: blood urea nitrogen; RRT: Renal replacement therapy; FST: furosemide stress test; IRB: institutional review board; KDIGO: Kidney Disease Improving Global Outcomes; MODS: multiple organ failure syndromes; CCU: coronary artery unit; CSRU: cardiac surgery recovery unit; MICU: medical ICU; SICU: surgical ICU; TSICU: trauma-neuro surgical ICU; AIC: Akaike Information Criterion; CI: Confidence interval; OR: Odds ratio; SHAP: SHapley Additive exPlanations

\section{Acknowledgements}

We would like to thank all the workers involved in MIMIC III database system maintenance. Especially to Aijia Ma and RuoRan Wang, for their help in data processing.

\section{Funding}

This project was supported by 1.3.5 project for disciplines of excellence, West China Hospital, Sichuan University (ZYGD18020).

Availability of data and materials

The datasets used for the analysis in the current study are available from the corresponding author on reasonable request.

Competing interests

The authors declare that they have no competing interests.

Author's contributions

LFZ, CZW and AJM designed the study. LFZ, CZW, JLC, XPZ and MLD managed data and its quality. LFZ and CZW performed the statistical analysis. All authors participated in data interpretation. LFZ drafted the manuscript. CZW, XPZ, AJM, JLC and MLD contributed substantially to its revision. All authors read the manuscript carefully and approved the final version.

\section{Ethics approval and consent to participate}

The study was an analysis of a third-party anonymized publicly available database with pre-existing institutional review board (IRB) approval.

Consent for publication

Not applicable.

\section{Author details}

${ }^{1}$ Department of Clinical Medical College, West China Hospital of Sichuan University, 610041 Chengdu, China.

${ }^{2}$ Department of Critical Care Medicine, West China Hospital of Sichuan University, 610041 Chengdu, China.

References

1. Group, K.D.I.G.O.K.A.K.I.W.: Kdigo clinical practice guideline for acute kidney injury. Kidney Int, 1-138 (2012)

2. Hoste, E.A.J., Kellum, J.A., Selby, N.M., Zarbock, A., Palevsky, P.M., Bagshaw, S.M., Goldstein, S.L., Cerda, J., Chawla, L.S.: Global epidemiology and outcomes of acute kidney injury. Nat Rev Nephrol 14(10), 607-625 (2018). doi:10.1038/s41581-018-0052-0

3. Chawla, L.S., Davison, D.L., Brasha-Mitchell, E., Koyner, J.L., Arthur, J.M., Shaw, A.D., Tumlin, J.A., Trevino, S.A., Kimmel, P.L., Seneff, M.G.: Development and standardization of a furosemide stress test to predict the severity of acute kidney injury. Crit Care 17(5), 207 (2013). doi:10.1186/cc13015

4. Deo, R.C.: Machine learning in medicine. Circulation 132(20), 1920-1930 (2015). doi:10.1161/CIRCULATIONAHA.115.001593

5. Johnson, A.E., Pollard, T.J., Shen, L., Lehman, L.W., Feng, M., Ghassemi, M., Moody, B., Szolovits, P., Celi, L.A., Mark, R.G.: Mimic-iii, a freely accessible critical care database. Sci Data 3, 160035 (2016). doi:10.1038/sdata.2016.35 
6. Lemaitre, G., Nogueira, F., Aridas, C.K.: Imbalanced-learn: A python toolbox to tackle the curse of imbalanced datasets in machine learning. Journal of Machine Learning Research 18 (2017)

7. Pozzoli, S., Simonini, M., Manunta, P.: Predicting acute kidney injury: current status and future challenges. J Nephrol 31(2), 209-223 (2018). doi:10.1007/s40620-017-0416-8

8. Wu, L., Hu, Y., Liu, X., Zhang, X., Chen, W., Yu, A.S.L., Kellum, J.A., Waitman, L.R., Liu, M.: Feature ranking in predictive models for hospital-acquired acute kidney injury. Sci Rep 8(1), 17298 (2018). doi:10.1038/s41598-018-35487-0

9. Isshiki, R., Asada, T., Sumida, M., Hamasaki, Y., Nangaku, M., Noiri, E., Doi, K.: Modest impact of serial measurements of acute kidney injury biomarkers in an adult intensive care unit. Nephron 139(3), 243-253 (2018). doi:10.1159/000488219

10. Pedregosa, F., Varoquaux, G., Gramfort, A., Michel, V., Thirion, B., Grisel, O., Blondel, M., Prettenhofer, P., Weiss, R., Dubourg, V., Vanderplas, J., Passos, A., Cournapeau, D., Brucher, M., Perrot, M.: Scikit-learn: Machine learning in python. Journal of Machine Learning Research 12(85), 2825-2830 (2011)

11. Stensrud, M.J., Hernan, M.A.: Why test for proportional hazards? JAMA 323(14), 1401-1402 (2020). doi:10.1001/jama.2020.1267

12. Chen, T., Guestrin, C.: Xgboost: A scalable tree boosting system. doi:10.1145/2939672.2939785

13. Tang, C.Q., Li, J.Q., Xu, D.Y., Liu, X.B., Hou, W.J., Lyu, K.Y., Xiao, S.C., Xia, Z.F.: Comparison of machine learning method and logistic regression model in prediction of acute kidney injury in severely burned patients. Zhonghua Shao Shang Za Zhi 34(6), 343-348 (2018). doi:10.3760/cma.j.issn.1009-2587.2018.06.006

14. Ferrari, F., Puci, M.V., Ferraro, O.E., Romero-Gonzalez, G., Husain-Syed, F., Rizo-Topete, L., Senzolo, M., Lorenzin, A., Muraro, E., Baracca, A., Serrano-Soto, M., Molano Trivino, A., Coutinho Castro, A., De Cal, M., Corradi, V., Brendolan, A., Scarpa, M., Carta, M.R., Giavarina, D., Bonato, R., lotti, G.A., Ronco, C.: Development and validation of quick acute kidney injury-score (q-aki) to predict acute kidney injury at admission to a multidisciplinary intensive care unit. PLoS One 14(6), 0217424 (2019). doi:10.1371/journal.pone.0217424

15. Lundberg, S.M., Lee, S.I.: A unified approach to interpreting model predictions. Advances in Neural Information Processing Systems 30 (Nips 2017) 30 (2017)

16. Lundberg, S.M., Nair, B., Vavilala, M.S., Horibe, M., Eisses, M.J., Adams, T., Liston, D.E., Low, D.K., Newman, S.F., Kim, J., Lee, S.I.: Explainable machine-learning predictions for the prevention of hypoxaemia during surgery. Nat Biomed Eng 2(10), 749-760 (2018). doi:10.1038/s41551-018-0304-0

17. Koyner, J.L., Carey, K.A., Edelson, D.P., Churpek, M.M.: The development of a machine learning inpatient acute kidney injury prediction model. Crit Care Med 46(7), 1070-1077 (2018). doi:10.1097/CCM.0000000000003123

18. Sanchez-Pinto, L.N., Venable, L.R., Fahrenbach, J., Churpek, M.M.: Comparison of variable selection methods for clinical predictive modeling. Int J Med Inform 116, 10-17 (2018). doi:10.1016/j.ijmedinf.2018.05.006

19. Zhang, Z., Ho, K.M., Hong, Y.: Machine learning for the prediction of volume responsiveness in patients with oliguric acute kidney injury in critical care. Crit Care 23(1), 112 (2019). doi:10.1186/s13054-019-2411-z

20. Zhang, Z.: Machine learning method for the management of acute kidney injury: more than just treating biomarkers individually. Biomark Med 13(15), 1251-1253 (2019). doi:10.2217/bmm-2019-0363

21. Kawamoto, K., Houlihan, C.A., Balas, E.A., Lobach, D.F.: Improving clinical practice using clinical decision support systems: a systematic review of trials to identify features critical to success. BMJ 330(7494), 765 (2005). doi:10.1136/bmj.38398.500764.8F

22. Wilson, F.P., Greenberg, J.H.: Acute kidney injury in real time: Prediction, alerts, and clinical decision support. Nephron 140(2), 116-119 (2018). doi:10.1159/000492064

23. Borasino, S., Wall, K.M., Crawford, J.H., Hock, K.M., Cleveland, D.C., Rahman, F., Martin, K.D., Alten, J.A.: Furosemide response predicts acute kidney injury after cardiac surgery in infants and neonates. Pediatr Crit Care Med 19(4), 310-317 (2018). doi:10.1097/PCC.0000000000001478

24. Ronco, C., Bellomo, R., Kellum, J.A.: Acute kidney injury. The Lancet 394(10212), 1949-1964 (2019). doi:10.1016/s0140-6736(19)32563-2

25. Flechet, M., Falini, S., Bonetti, C., Guiza, F., Schetz, M., Van den Berghe, G., Meyfroidt, G.: Machine learning versus physicians' prediction of acute kidney injury in critically ill adults: a prospective evaluation of the akipredictor. Crit Care 23(1), 282 (2019). doi:10.1186/s13054-019-2563-x

26. Bhatraju, P.K., Zelnick, L.R., Katz, R., Mikacenic, C., Kosamo, S., Hahn, W.O., Dmyterko, V., Kestenbaum, B., Christiani, D.C., Liles, W.C., Himmelfarb, J., Wurfel, M.M.: A prediction model for severe aki in critically il adults that incorporates clinical and biomarker data. Clin J Am Soc Nephrol 14(4), 506-514 (2019). doi:10.2215/CJN.04100318

27. Koyner, J.L., Davison, D.L., Brasha-Mitchell, E., Chalikonda, D.M., Arthur, J.M., Shaw, A.D., Tumlin, J.A., Trevino, S.A., Bennett, M.R., Kimmel, P.L., Seneff, M.G., Chawla, L.S.: Furosemide stress test and biomarkers for the prediction of aki severity. J Am Soc Nephrol 26(8), 2023-31 (2015). doi:10.1681/ASN.2014060535

Figures

Figure 1 Flow chart of patient selection

Figure 2 Cox regression model with stepwise variable selection The odds ratio [OR], and confidence interval are shown in the horizontal axis. $\mathrm{p}<0.05, * ; \mathrm{p}<0.01, * * ; \mathrm{p}<0.001$, *** 
Figure 3 The training process of the extreme gradient boosting machine A: Cross-validation during $X$ GBoost hyperparameter tuning. The log-loss value for the training and testing datasets is shown in the vertical axis. The dashed vertical line indicates the number of rounds with the minimum log-loss in the test sample. B: Learning curve of the XGBoost model after hyperparameter tuning. AU-ROC value for the testing and training datasets is shown in the vertical axis. With the subsample ratio increasing, AU-ROC of training datasets decreases, and AU-ROC of testing datasets increases. The training score is always higher than the test score

Figure 4 Performance of the XGBoost and Cox regression model A: Receiver operating characteristic curve for estimating the discrimination between the Cox regression model and the XGBoost model. The true positive rate and false positive rate are shown in the vertical axis and horizontal axis respectively. B: Confusion matrix of the Cox regression model and XGBoost model. The color represents the number of patients. Whether progress is represented by numbers.

Figure 5 SHAP value of XGBoost model output A: SHAP value of one patient output. The contribution and value of every variable are shown. The longer the color bar, the greater contribution in the corresponding direction. The output pf the model id obtained by calculating the sum of all variables' contributions based on the base value. B: SHAP value of all patient output. Each point represents a variable for an observation. The color of the point is determined by its relative height in the variable. The blue represents lower and the red represents higher.

Figure 6 Feature importance derived from the XGBoost model Feature importance was calculated by the mean contribution of every observation, which is equal to the traditional method. Abbreviations and annotations: BUN, blood urea nitrogen; FST, furosemide stress test; MODS, multiple organ failure syndromes

Figure 7 SHAP value for single variable and interaction A: SHAP value for creatinine. SHAP value increases with the increase of creatinine until creatinine probably reaches $5 \mathrm{mg} / \mathrm{dL}$. B: SHAP value for FST. SHAP value decreases with the increase of FST until FST reach $100 \mathrm{ml} / \mathrm{h}$. C: SHAP value for interaction on age and creatinine. The color of the point represents the value of creatinine. The SHAP value is extremely high in patients who are older than 70 , only when creatinine $>3.0 \mathrm{mg} / \mathrm{dl}$ 
Table 1 Characteristics between progression and non-progression groups

\begin{tabular}{|c|c|c|c|c|}
\hline Variables & Overall & Non-progression $(n=24329)$ & Progression $(n=3119)$ & P-value \\
\hline \multicolumn{5}{|l|}{ Gender, n (\%) } \\
\hline Female & $11413(41.6)$ & $10054(41.3)$ & $1359(43.6)$ & 0.017 \\
\hline Male & $16035(58.4)$ & $14275(58.7)$ & $1760(56.4)$ & \\
\hline \multirow{2}{*}{\multicolumn{5}{|c|}{$\begin{array}{l}\text { Age, mearan [Q1,QS] } \\
\text { Ethnicity, n (\%) }\end{array}$}} \\
\hline & & & & \\
\hline Asian & $555(2.0)$ & $497(2.0)$ & $58(1.9)$ & $<0.001$ \\
\hline Black & $2536(9.2)$ & $2072(8.5)$ & $464(14.9)$ & \\
\hline Hispanic & $809(2.9)$ & $686(2.8)$ & $123(3.9)$ & \\
\hline Native & $14(0.1)$ & $10(0.0)$ & $4(0.1)$ & \\
\hline Other & $706(2.6)$ & $628(2.6)$ & $78(2.5)$ & \\
\hline Unknown & $3199(11.7)$ & $2857(11.7)$ & $342(11.0)$ & \\
\hline White & $19629(71.5)$ & $17579(72.3)$ & $2050(65.7)$ & \\
\hline \multicolumn{5}{|l|}{ Admissions type, $\mathrm{n}(\%)$} \\
\hline Elective & $4616(16.8)$ & $4384(18.0)$ & $232(7.4)$ & $<0.001$ \\
\hline Emergency & $22047(80.3)$ & $19253(79.1)$ & $2794(89.6)$ & \\
\hline Urgent & $785(2.9)$ & $692(2.8)$ & $93(3.0)$ & \\
\hline \multicolumn{5}{|l|}{ ICU type, n (\%) } \\
\hline $\mathrm{CCU}$ & $3905(14.2)$ & $3407(14.0)$ & $498(16.0)$ & $<0.001$ \\
\hline CSRU & $6675(24.3)$ & $6298(25.9)$ & $377(12.1)$ & \\
\hline MICU & 9567 (34.9) & $8116(33.4)$ & $1451(46.5)$ & \\
\hline SICU & $4247(15.5)$ & 3678 (15.1) & $569(18.2)$ & \\
\hline TSICU & $3054(11.1)$ & $2830(11.6)$ & $224(7.2)$ & \\
\hline Cardiac surgery, n (\%) & $21926(79.9)$ & $19671(80.9)$ & $2255(72.3)$ & $<0.001$ \\
\hline Respiratory failure, n (\%) & $10116(36.9)$ & $8219(33.8)$ & $1897(60.8)$ & $<0.001$ \\
\hline Mechanical ventilation, $\mathrm{n}(\%)$ & $7738(30.9)$ & $6752(29.6)$ & $986(43.6)$ & $<0.001$ \\
\hline MODS, n (\%) & $17692(64.5)$ & $14706(60.4)$ & $2986(95.7)$ & $<0.001$ \\
\hline Sepsis, $\mathrm{n}(\%)$ & $4949(18.0)$ & $3648(15.0)$ & $1301(41.7)$ & $<0.001$ \\
\hline Vasoactive drugs, $\mathrm{n}(\%)$ & $8496(31.0)$ & $7573(31.1)$ & $923(29.6)$ & 0.085 \\
\hline \multicolumn{5}{|l|}{ Laboratory variables, mean (SD) } \\
\hline $\mathrm{AaDO} 2(\mathrm{mmHg})$ & $438.5(127.8)$ & $435.2(127.9)$ & $459.2(125.1)$ & $<0.001$ \\
\hline Anion gap $(\mathrm{mEg} / \mathrm{l})$ & $13.7(3.8)$ & $13.4(3.6)$ & $15.8(4.5)$ & $<0.001$ \\
\hline BUN (mg/dl) & $26.3(20.1)$ & $24.5(18.3)$ & $41.3(27.1)$ & $<0.001$ \\
\hline Creatinine $(\mathrm{mg} / \mathrm{dl})$ & $1.4(1.5)$ & $1.3(1.3)$ & $2.9(2.4)$ & $<0.001$ \\
\hline FST $(\mathrm{ml} / \mathrm{h})$ & $194.7(146.8)$ & $198.5(134.3)$ & $158.6(231.2)$ & 0.001 \\
\hline Glucose $(\mathrm{mg} / \mathrm{dl})$ & $136.9(72.2)$ & $136.4(68.9)$ & $140.7(94.4)$ & 0.014 \\
\hline Lactate $(\mathrm{mmol} / \mathrm{I})$ & $2.1(1.7)$ & $2.0(1.6)$ & $2.4(2.1)$ & $<0.001$ \\
\hline $\mathrm{PaO} 2(\mathrm{mmHg})$ & $127.8(78.3)$ & $128.3(77.9)$ & $124.4(81.6)$ & 0.035 \\
\hline $\mathrm{pH}$ & $7.4(0.1)$ & $7.4(0.1)$ & $7.4(0.1)$ & $<0.001$ \\
\hline Potassium (mmol/l) & $4.2(0.7)$ & $4.2(0.6)$ & $4.4(0.8)$ & $<0.001$ \\
\hline $\begin{array}{l}\text { Sodium (mmol/l) } \\
\text { vital signs, mean (SD) }\end{array}$ & $138.4(4.7)$ & $138.5(4.6)$ & $137.7(5.3)$ & $<0.001$ \\
\hline Blood pressure (mmHg) & $76.7(16.3)$ & $76.8(16.1)$ & $76.2(18.7)$ & 0.14 \\
\hline Heart rate & $86.0(18.3)$ & $85.7(18.0)$ & $89.4(20.7)$ & $<0.001$ \\
\hline Respiratory rate & $19.1(6.0)$ & $19.0(5.9)$ & $20.0(6.6)$ & $<0.001$ \\
\hline Temperature $\left({ }^{\circ} \mathrm{C}\right)$ & $36.9(0.6)$ & $36.9(0.6)$ & $36.8(0.7)$ & $<0.001$ \\
\hline
\end{tabular}

Table 2 Cox regression model with stepwise variable selection

\begin{tabular}{llll}
\hline Variables & OR & $95 \% \mathrm{Cl}$ & P-value \\
\hline Age & 1.00 & $1.00,1.01$ & $<0.001$ \\
Blood pressure & 1.00 & $0.99,1.00$ & $<0.001$ \\
Heart rate & 1.00 & $1.00,1.01$ & $<0.001$ \\
Respiratory rate & 1.00 & $1.00,1.01$ & 0.38 \\
Temperature & 0.84 & $0.81,0.86$ & $<0.001$ \\
Cardiac surgery & 1.09 & $1.03,1.15$ & $<0.001$ \\
Mechanical ventilation & 1.22 & $1.16,1.29$ & $<0.001$ \\
MODS & 2.90 & $2.68,3.15$ & $<0.001$ \\
Respiratory failure & 1.21 & $1.15,1.28$ & $<0.001$ \\
Sepsis & 1.56 & $1.49,1.63$ & $<0.001$ \\
Vasoactive drugs & 1.09 & $1.04,1.14$ & $<0.001$ \\
AaDO2 & 1.00 & $1.00,1.00$ & $<0.001$ \\
Anion gap & 1.01 & $1.01,1.02$ & $<0.001$ \\
BUN & 1.00 & $1.00,1.00$ & 0.02 \\
Creatinine & 1.17 & $1.16,1.18$ & $<0.001$ \\
Glucose & 1.00 & $1.00,1.00$ & 0.31 \\
Lactate & 1.03 & $1.01,1.04$ & $<0.001$ \\
PaO2 & 1.00 & $1.00,1.00$ & 0.44 \\
pH & 0.61 & $0.47,0.80$ & $<0.001$ \\
Potassium & 1.05 & $1.02,1.09$ & $<0.001$ \\
Sodium & 0.99 & $0.98,0.99$ & $<0.001$ \\
\hline
\end{tabular}


Figures

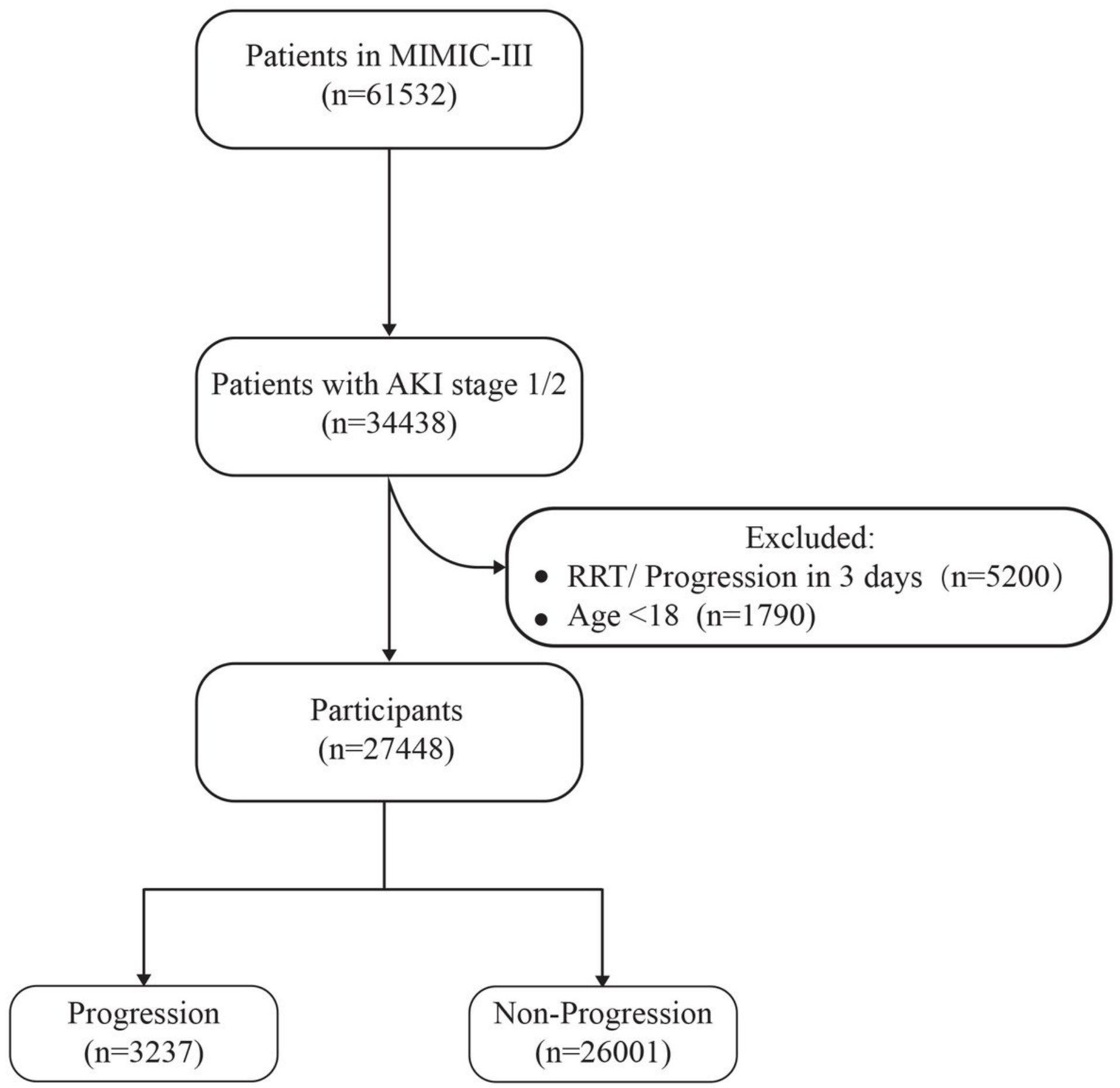

Figure 1

Flow chart of patient selection 


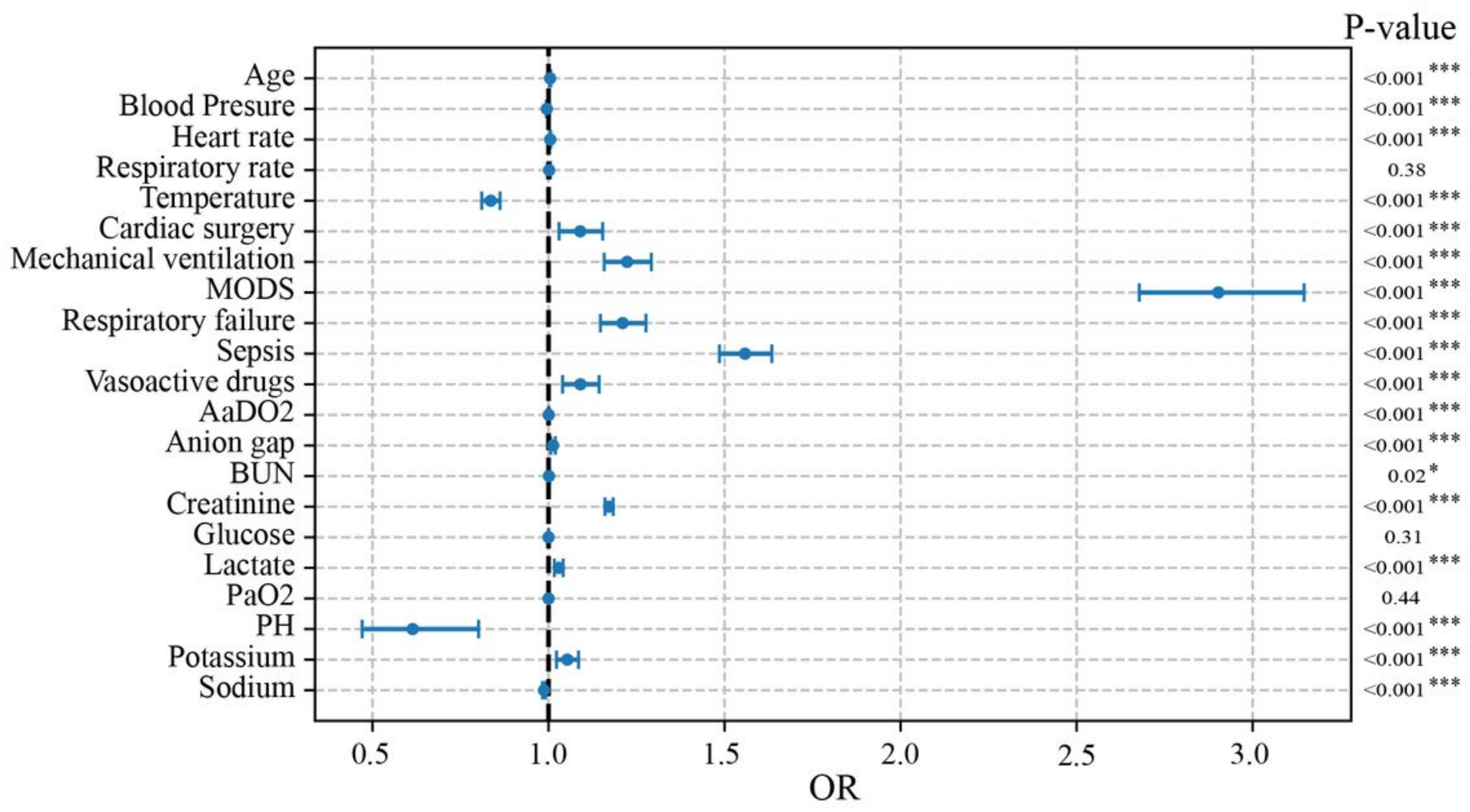

Figure 2

Cox regression model with stepwise variable selection The odds ratio [OR], and confidence interval are shown in the horizontal axis. $p<0.05, * ; p<0.01, * * ; p<0.001, * \star \star$ 
A

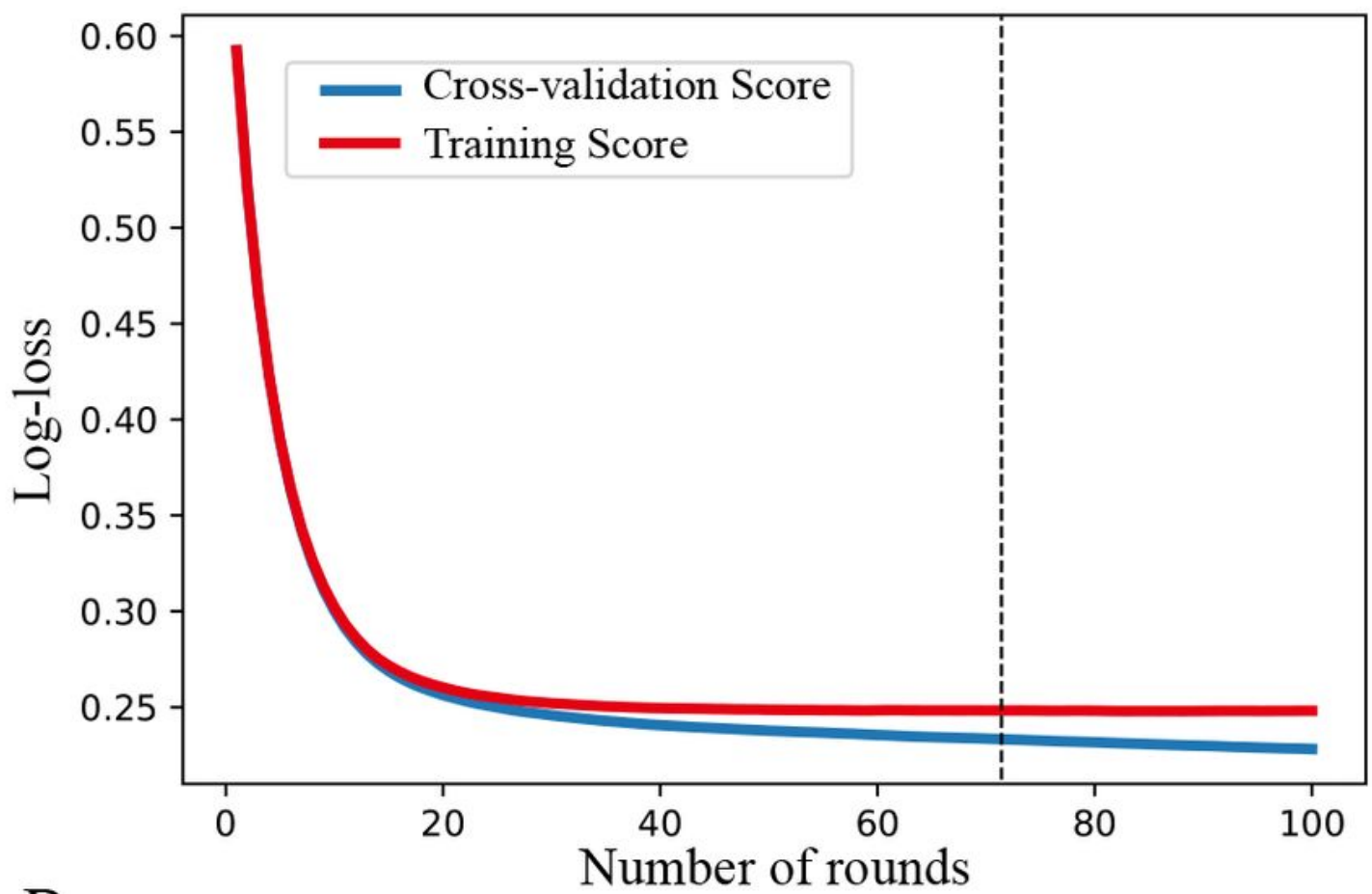

B

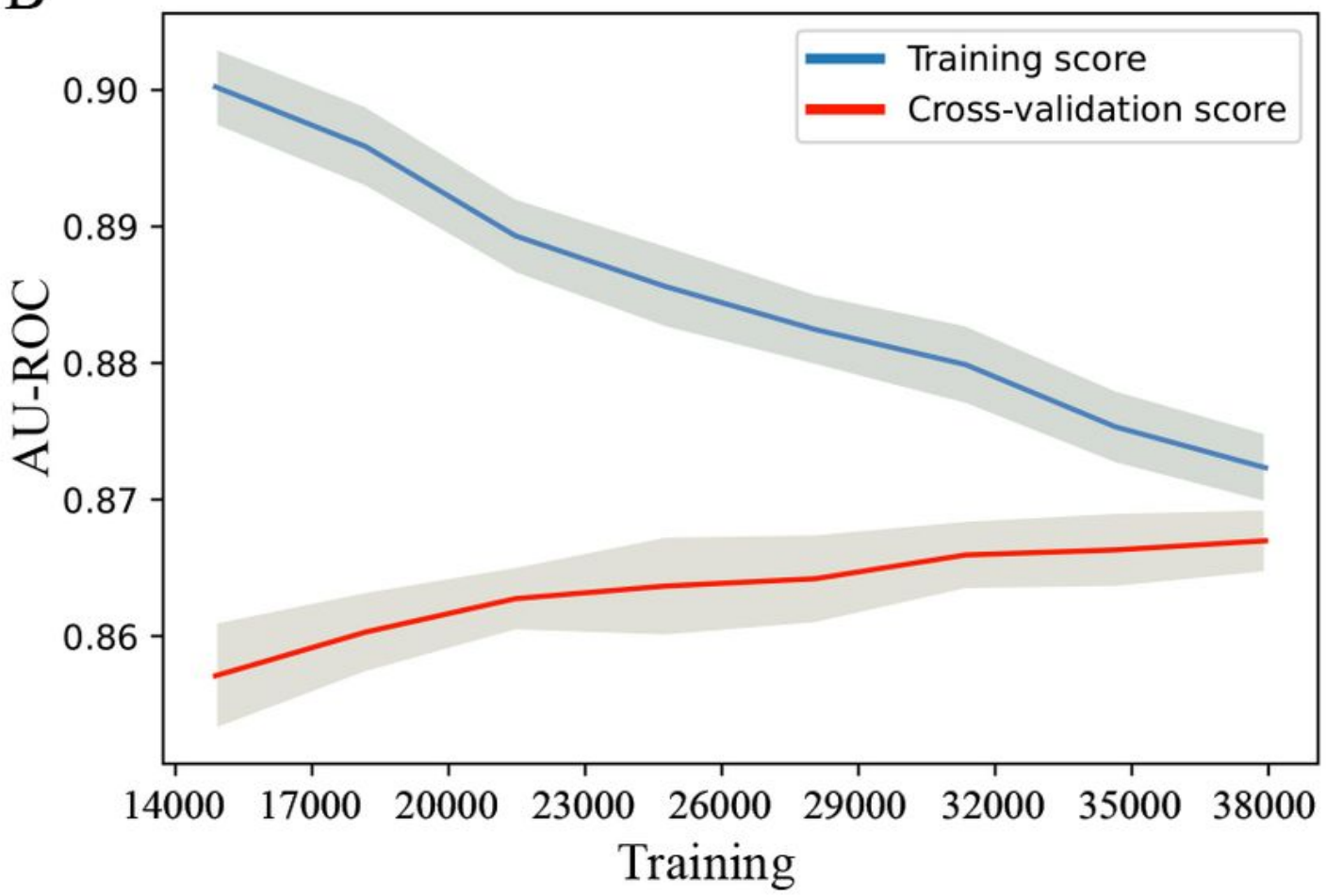

Figure 3

The training process of the extreme gradient boosting machine A: Cross-validation during XGBoost hyperparameter tuning. The log-loss value for the training and testing datasets is shown in the vertical axis. The dashed vertical line indicates the number of rounds with the minimum log-loss in the test sample. B: Learning curve of the XGBoost model after hyperparameter tuning. AU-ROC value for the testing and training datasets is shown in the vertical axis. With the subsample ratio increasing, AU-ROC of 
training datasets decreases, and AU-ROC of testing datasets increases. The training score is always higher than the test score

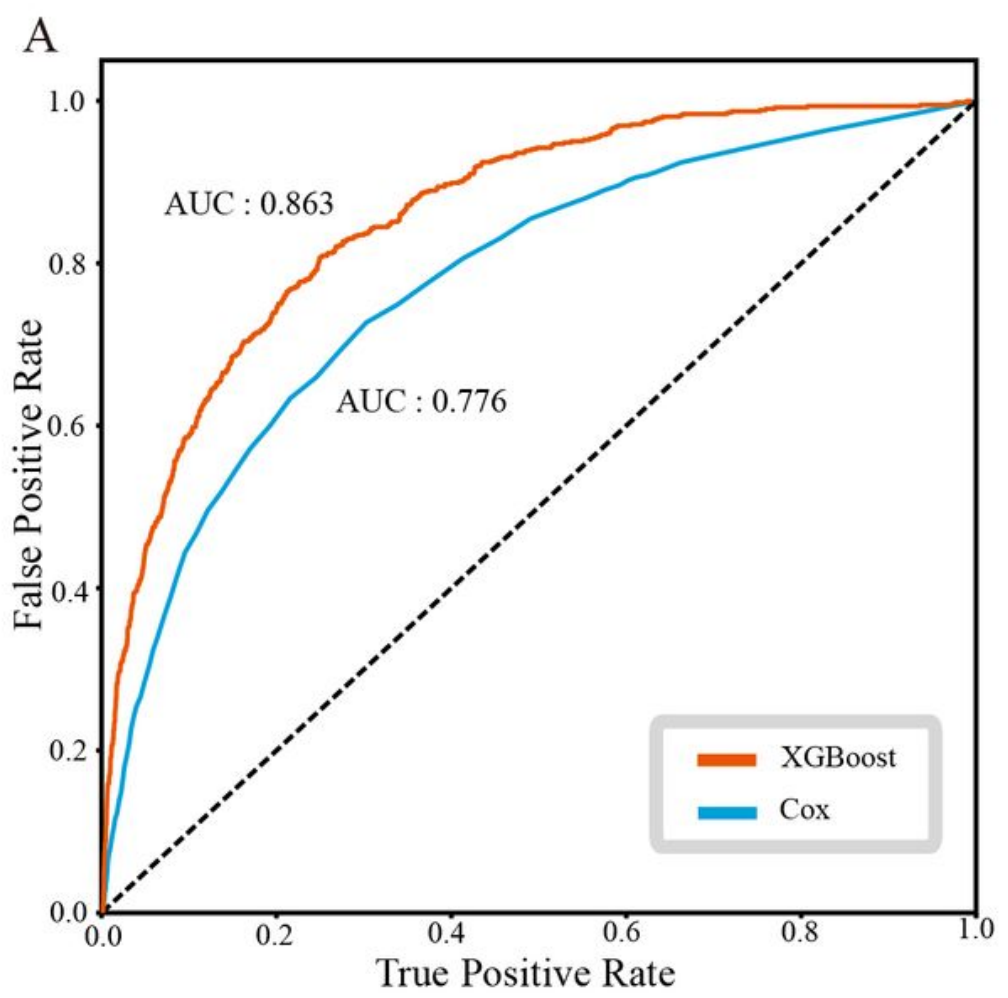

B

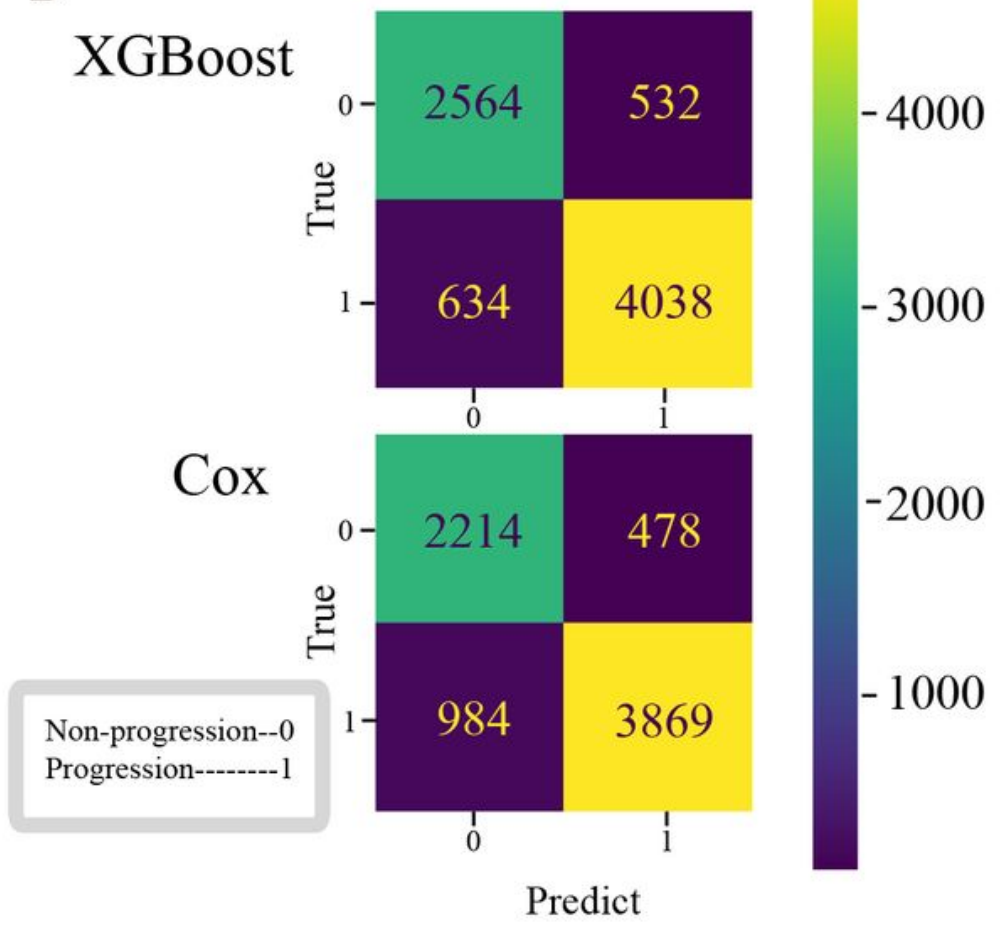

Figure 4

Performance of the XGBoost and Cox regression model A: Receiver operating characteristic curve for estimating the discrimination between the Cox regression model and the XGBoost model. The true positive rate and false positive rate are shown in the vertical axis and horizontal axis respectively. B: 
Confusion matrix of the Cox regression model and XGBoost model. The color represents the number of patients. Whether progress is represented by numbers.

A

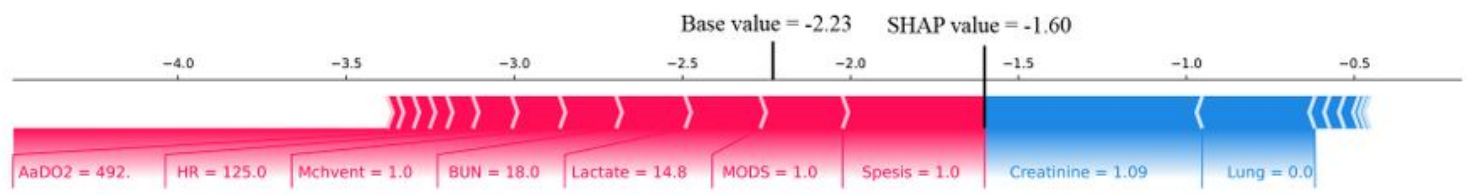

B

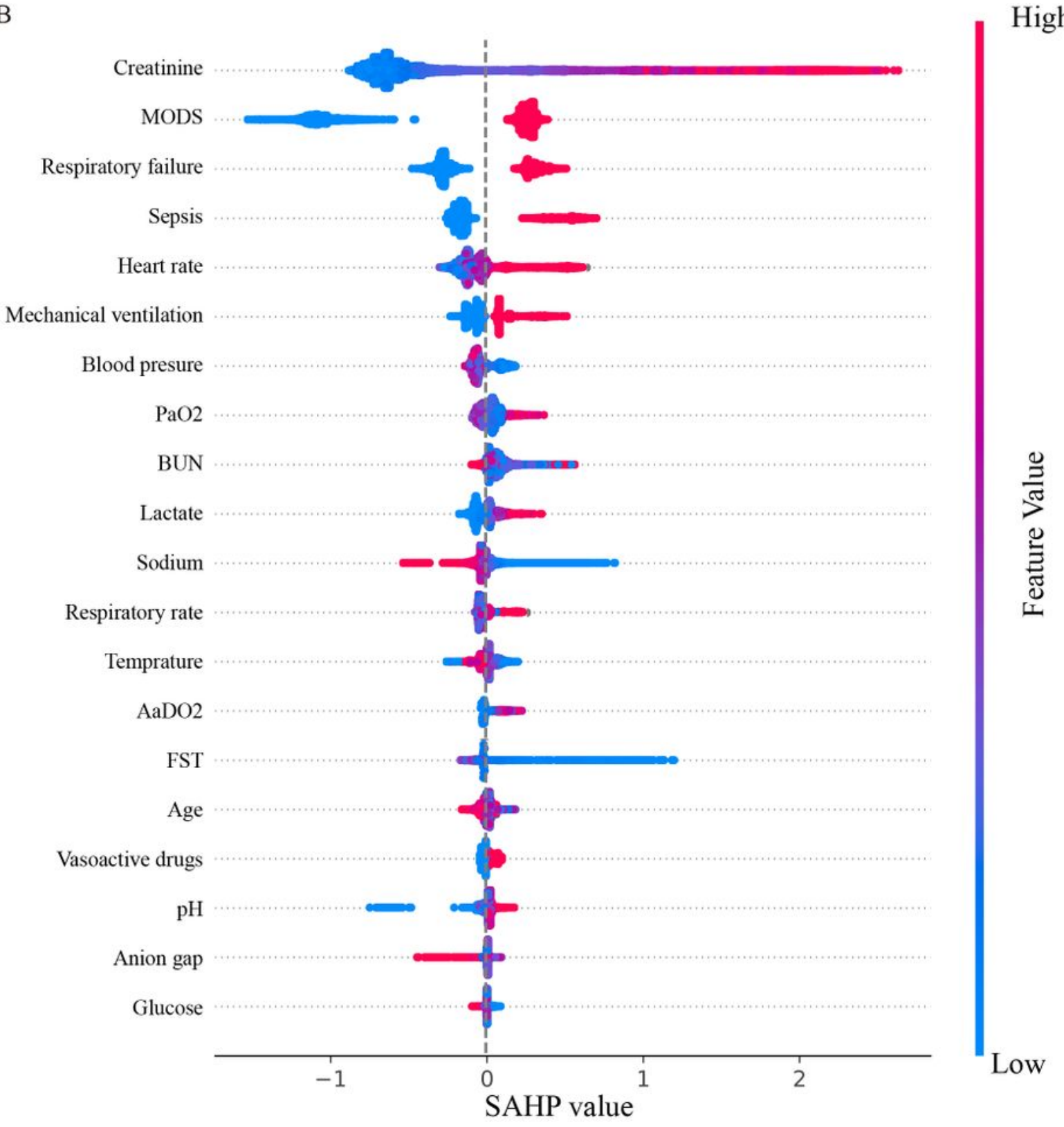

Figure 5

SHAP value of XGBoost model output A: SHAP value of one patient output. The contribution and value of every variable are shown. The longer the color bar, the greater contribution in the corresponding direction. The output pf the model id obtained by calculating the sum of all variables' contributions based on the 
base value. B: SHAP value of all patient output. Each point represents a variable for an observation. The color of the point is determined by its relative height in the variable. The blue represents lower and the red represents higher.

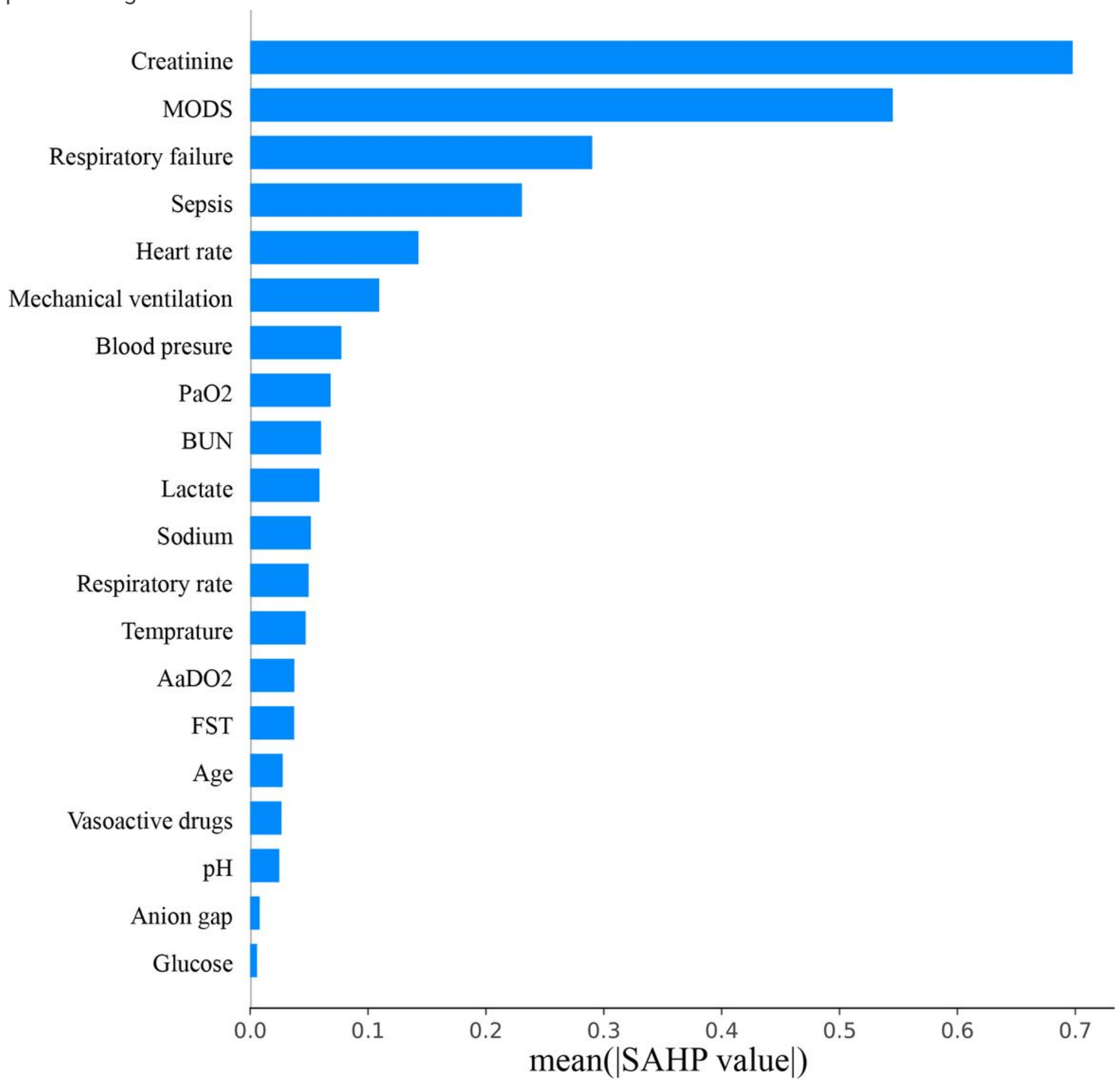

\section{Figure 6}

Feature importance derived from the XGBoost model Feature importance was calculated by the mean contribution of every observation, which is equal to the traditional method. Abbreviations and annotations: BUN, blood urea nitrogen; FST, furosemide stress test; MODS, multiple organ failure syndromes 

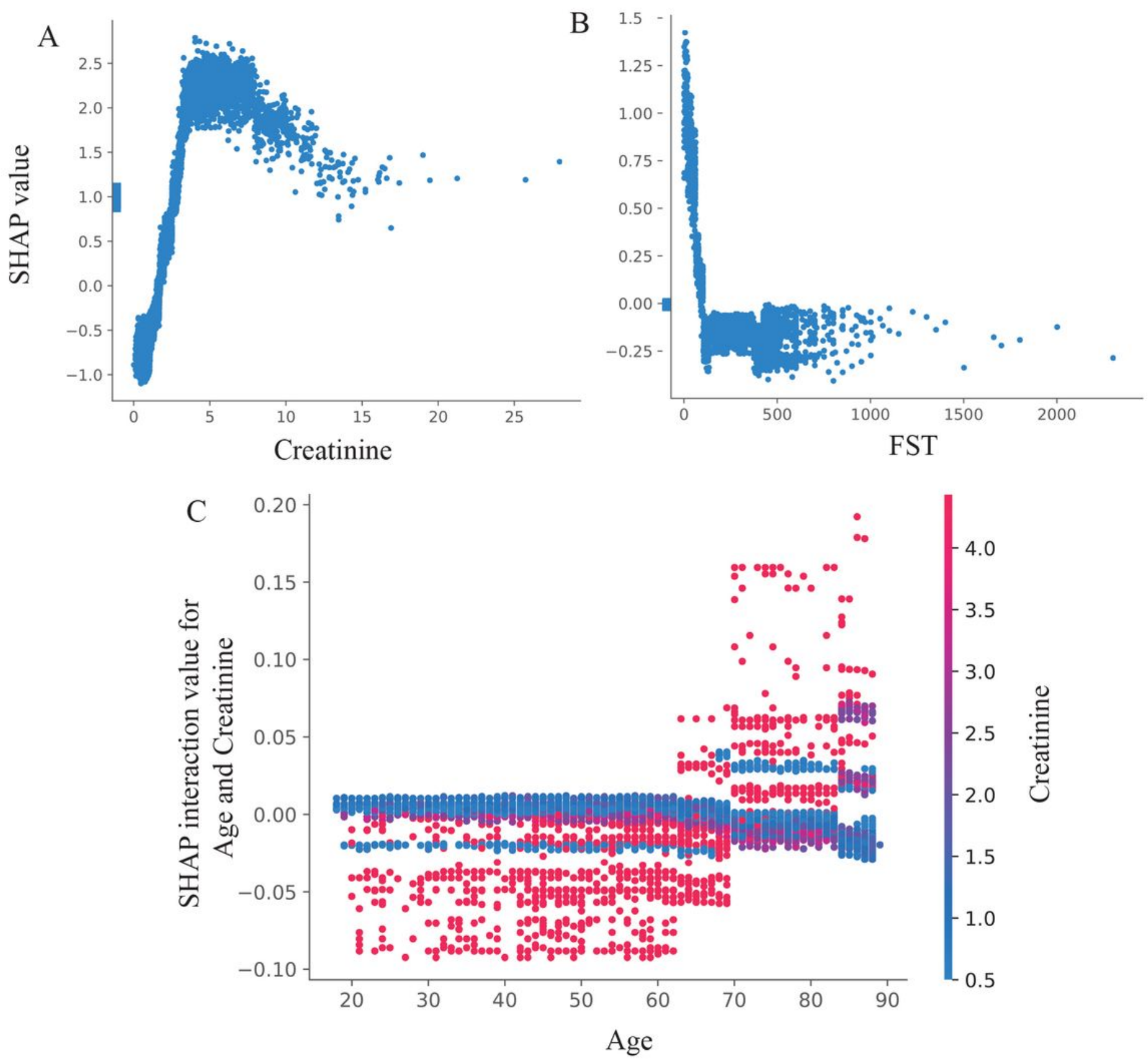

Figure 7

SHAP value for single variable and interaction A: SHAP value for creatinine. SHAP value increases with the increase of creatinine until creatinine probably reaches $5 \mathrm{mg} / \mathrm{dL}$. B: SHAP value for FST. SHAP value decreases with the increase of FST until FST reach $100 \mathrm{ml} / \mathrm{h}$. C: SHAP value for interaction on age and creatinine. The color of the point represents the value of creatinine. The SHAP value is extremely high in patients who are older than 70 , only when creatinine $>3.0 \mathrm{mg} / \mathrm{dl}$ 